Théologiques

Théologiques

\title{
Des voyageurs immobiles. Pratiques communautaires autour du pèlerinage (Haute Égypte)
}

\section{Frédérique Fogel}

Volume 15, numéro 1, 2007

Mémoires et usages religieux de l'espace

URI : https://id.erudit.org/iderudit/017630ar

DOI : https://doi.org/10.7202/017630ar

Aller au sommaire du numéro

\section{Éditeur(s)}

Faculté de théologie et de sciences des religions, Université de Montréal

\section{ISSN}

1188-7109 (imprimé)

1492-1413 (numérique)

Découvrir la revue

\section{Citer cet article}

Fogel, F. (2007). Des voyageurs immobiles. Pratiques communautaires autour du pèlerinage (Haute Égypte). Théologiques, 15(1), 19-42.

https://doi.org/10.7202/017630ar
Résumé de l'article

Cet article aborde le rite de pèlerinage majeur de l'islam, le grand pèlerinage à La Mecque, par l'observation conjointe des dimensions individuelle et collective. Celui qui accomplit le hajj connaît une expérience unique dont il revient transformé. La communauté villageoise facilite son départ, vit le rituel par procuration, réintègre le pèlerin à son retour. En son absence, les " voyageurs immobiles » ornent de peintures la façade de sa maison, célébrant l'acte de piété, exposant leurs valeurs, affichant le pacte social. L'analyse des engagements individuel et collectif fait apparaître que la logique du rite de passage est plurielle, relative aux configurations multiples de la présence et de l'absence, du rôle et du statut, du temps et de l'espace, de l'expérience et de l'émotion.
Tous droits réservés @ Faculté de théologie et de sciences des religions, Université de Montréal, 2007
Ce document est protégé par la loi sur le droit d'auteur. L'utilisation des services d'Érudit (y compris la reproduction) est assujettie à sa politique d'utilisation que vous pouvez consulter en ligne.

https://apropos.erudit.org/fr/usagers/politique-dutilisation/ 


\title{
Des voyageurs immobiles. Pratiques communautaires autour du pèlerinage (Haute Égypte)
}

\author{
Frédérique FogEL \\ Laboratoire d'ethnologie et de sociologie comparative \\ Université de Paris X, Nanterre
}

Le hajj, «grand pèlerinage » à La Mecque, constitue l'un des cinq « piliers » (arkân) de l'islam, prescriptions fondatrices et essentielles ${ }^{1}$. Tout musulman doit faire ce pèlerinage une fois dans sa vie adulte, s'il en a les moyens physiques et matériels. Chaque année, les cérémonies rituelles du hajj se déroulent à La Mecque et dans les environs, du 8 au 12 du mois dhu el-hijja ( "mois du pèlerinage », douzième du calendrier hégirien), et nécessitent un voyage de deux à six semaines selon le lieu de résidence des croyants et leur choix de visites complémentaires à Médine. Décision, préparation, départ, réalisation, retour, chaque étape présente d'abord un versant individuel et volontaire, et le bon accomplissement de ce rite de passage fera d'un croyant ordinaire un hâjj, terme qui désigne celui qui a accompli le grand pèlerinage. Cette nouvelle identité religieuse donne accès à un nouveau statut social, diversement valorisé selon les pays et les régions du monde musulman. Les femmes, quels que soient leur âge et leur condition, sont accompagnées par un homme appartenant à leur parenté. Le pèlerin est également, dans le même temps, le personnage central d'une aventure collective, à laquelle participent ses parents, ses proches, ses voisins, ses collègues et ses amis. Dans l'Égypte rurale, en Haute Égypte et en Nubie égyptienne, le pèlerinage d'un seul concerne et engage tous les membres de sa communauté.

En effet, ceux qui ne partent pas participent à la décision, contribuent à la préparation et au financement du voyage, organisent des fêtes pour le

1. Avec la profession de foi, les cinq prières quotidiennes, l'aumône, le jeûne de ramadan. Le grand pèlerinage se distingue du petit pèlerinage ('umra), visite à la Ka'aba effectuée à n'importe quelle autre période de l'année. 
départ et le retour des pèlerins. Pendant le hajj, ils vivent «à l'heure de » La Mecque, passent des soirées entières à raconter leur propre pèlerinage ou celui qu'ils feront bientôt, in shâa' Allah («si Dieu le veut »). Ils suivent quotidiennement le déroulement du rituel grâce aux émissions religieuses et aux reportages de la radio et de la télévision. Ils s'inquiètent des accidents de transport et des attentats politiques, des mouvements de foule qui laissent des victimes piétinées, et se rassurent en répétant que «celui qui meurt en pèlerinage ira au paradis ». Au matin du $10 \mathrm{dhu}$ el-hijja, ils procèdent au sacrifice d'un animal, en union avec les pèlerins dans la vallée de Mina et avec l'ensemble du monde musulman. Sauf si le pèlerin y est opposé et l'a explicitement interdit avant son départ, ils se réunissent pour décorer d'images et de textes la façade de sa maison. À son retour, ils recueillent la baraka ${ }^{2}$ contenue dans son récit, dans les objets et la nourriture qu'il a rapportés pour leur offrir, et dans son nouveau statut. Sans se déplacer, ils participent au pèlerinage : ce sont les voyageurs immobiles.

Cet article tente un décentrement épistémologique du regard anthropologique sur le rite du pèlerinage. Les sciences sociales traitent généralement la problématique complexe du pèlerinage en focalisant sur le rite l'expérience spirituelle, le rite de passage —, sur l'acteur central et, plus récemment, sur l'organisation, les organisateurs et les effets du voyage dans l'environnement socioculturel du lieu de pèlerinage ${ }^{3}$. Je propose de porter l'attention sur les interactions entre l'individuel et le collectif dans les rites de passage particuliers que sont les pèlerinages, en prenant aussi en compte le lieu de départ, c'est-à-dire le lieu de vie habituel des pèlerins. À tout le moins, le hajj est une expérience individuelle unique en ce qu'elle ne transforme pas seulement l'acteur principal : ceux qui ne partent pas vivent par procuration le voyage pieux d'un des leurs. Plus encore, ils jouent la partie collective d'un rite qui les transforme, à l'image du collectif des pèlerins accomplissant les rituels à La Mecque. Cette transformation multiforme a une caractéristique: son personnage principal est absent, et c'est précisément son absence qui rend possible le voyage immobile des membres de sa communauté - voyage immobile, voyage imaginaire très concret, et assez agité.

2. Ce terme, qui signifie littéralement "bénédiction ", désigne une "force bénéfique » d'origine divine, s'exprimant notamment par la médiation des saints qui la dispensent aux croyants, et à travers des objets ou des lieux considérés comme sacrés.

3. Voir notamment le programme de recherche "Tourisme et culture religieuse au Maghreb. Approches anthropologiques », dirigé par Katia Boissevain à l'Institut de Recherche sur le Maghreb Contemporain (Tunis). 


\section{Se préparer à partir}

Une grande part de la préparation au pèlerinage est d'ordre spirituel. Comme le rappelle Al-Ghazali, le fidèle doit rompre ses attaches avec le monde et se préparer comme s'il n'allait pas en revenir (Bousquet 1955). Ce qui signifie régler ses affaires, prendre ses dispositions, résoudre le cas échéant les conflits familiaux, sociaux, professionnels dans lesquels on se trouve engagé. Il faut pouvoir répondre à l'appel de Dieu sans pénaliser ses proches: pour un homme chargé de famille, assurer l'avenir des siens fait partie des moyens requis pour accomplir le hajj. Se détacher des contingences matérielles et se mettre en paix avec chacun — et avec soi-même permet de se préparer à sortir, libre, de la communauté. Il s'agit donc, à mon avis, tout autant de la première phase de tout rite de passage, qui consiste en ce dénuement qui prélude à l'exclusion, que de l'anticipation, par le pèlerinage, du retour à Dieu que le croyant accomplira à sa mort. L'écho émotionnel de cette mise en disposition est perceptible chez l'individu comme chez ses proches.

Cette dimension sensible indique également un aspect notable de la préparation du pèlerinage: si l'injonction est une adresse individuelle, la décision relève le plus souvent d'une sphère collective inscrite dans la parenté et relevant des modalités de relation entre les générations. Par exemple, les enfants d'âge adulte encouragent leurs parents. Bien que le bon accomplissement du rite nécessite que chacun finance le voyage par lui-même, les proches participent si besoin est. L'âge relatif des pèlerins varie selon leurs ressources et leur capital culturel, qui découle de leur statut social. Pour les plus modestes, le financement est l'affaire d'une longue période; le fils d'un hâij pauvre ne prévoit pas faire le pèlerinage du vivant de son père. Dans les milieux plus aisés, il est fréquent que le père de famille effectue un premier pèlerinage seul, puis un second avec son épouse; il est possible aussi que des membres de deux générations successives s'y engagent dans une même période. En dépit d'une volonté personnelle explicite, certains jeunes adultes sont jugés trop immatures pour partir, même s'ils sont déjà mariés et pères de famille4. Dans le cas où un fils — aîné ou cadet, le rang n'importe pas - accompagne sa mère veuve, il agit alors en tant que

4. Pour les émigrés qui travaillent en Arabie saoudite ou dans les pays du Golfe, la proximité géographique et les commodités administratives influencent certainement la décision, ce qui peut modifier la norme sociale du lieu d'origine, dans une certaine mesure. 
chef de famille, le statut prévalant sur l'âge, voire sur l'expérience. Il reste que, en règle générale, un homme jeune fait le pèlerinage pour lui-même alors qu'un homme accompli le fait aussi pour sa famille, et plus largement pour sa communauté. Comme tout rite de passage exécuté au moment opportun, le pèlerinage ne fait que confirmer, vis-à-vis du statut du pèlerin, une potentialité arrivée à maturité, et jugée comme telle par les membres de la communauté. Les voyageurs immobiles s'impliquent concrètement dans l'aventure.

Les proches du futur hâij l'aident à rassembler l'argent nécessaire au voyage, puis à obtenir l'agrément des autorités nationales. Chaque pays musulman dispose d'un quota annuel décidé par l'Arabie saoudite en fonction des données démographiques. Le pèlerin peut choisir entre deux modes d'organisation. Le système privé, dit siyahi ( «touristique »), est entièrement géré par les agences de voyages qui effectuent les démarches administratives et offrent plus de confort dans les déplacements et l'hébergement; le système du nizâm al-qera'a ( "tirage au sort») désigne les bénéficiaires d'une aide financière de l'État qui organisent leur voyage eux-mêmes, indépendamment ou en groupe, en utilisant les renseignements fournis par ceux qui les ont précédés. En Égypte, hommes et femmes s'inscrivent auprès du commissariat de leur district. Certains candidats peuvent être soutenus par des notables locaux. Deux mois environ avant le départ, la liste des noms tirés au sort est publiée dans la presse régionale. Il faut alors constituer un dossier comprenant des renseignements sur la famille, sur les moyens financiers, et des certificats de vaccination. L'administration établit un passeport «de pèlerinage », imprimé en arabe, en français et en anglais. Le document, valable trois mois, porte les dates du départ et du retour, et mentionne le montant du dépôt que le futur pèlerin a effectué à la banque nationale de son pays. En échange, il reçoit un chèque convertible à l'arrivée en monnaie saoudienne.

\section{Pèleriner}

Le voyage débute au village, quand le futur hâjj sort de sa maison et s'installe à la place d'honneur dans la voiture ou le minibus décoré de palmes et de drapeaux blancs et verts. La famille et les amis s'entassent à l'arrière, et c'est un cortège criant, chantant et klaxonnant qui l'accompagne jusqu'au lieu de regroupement régional. Dans la gare, le stade ou l'aéroport, un véritable campement s'établit, et va se maintenir plusieurs jours, car l'heure de la convocation n'indique en rien celle du départ. Des marchands 
de thé et de soda, des musiciens de mizmar ( «hautbois») et de tabla («tambourin ») proposent leurs services. Des hommes dansent et défilent derrière leur représentant; des femmes chantent entre elles. L'excitation du départ est amplement partagée, la joie cédant la place à l'émotion quand le voyageur franchit la barrière. Jusqu'à ce moment, il avait lentement construit sa distinction au milieu de ses parents, de ses proches. La communauté l'a soutenu dans son projet, l'aidant à se détacher d'elle, à en sortir.

Maintenant, il quitte les siens et la foule de ceux qui restent, et rejoint pour y prendre sa place un autre groupe, celui de ses semblables, ses frères et sœurs de pèlerinage. Le voyage devient synonyme d'attente et de patience, qualité essentielle requise pour le bon accomplissement du rite. Les pèlerins forment une foule impressionnante, canalisée par les forces de l'ordre dans le dédale des contrôles, formalités douanières, inspections des bagages. À l'aller comme au retour, le statut religieux de ces voyageurs particuliers ne leur vaut aucun privilège. En avion ou en bateau, la distance est courte, mais le temps passe lentement, à la mesure des dispositifs d'accueil dans les ports et les aéroports, toujours débordés par le nombre des participants. À Djedda, les pèlerins sont pris en charge par des guides (mutawwiffin, sing. mutawwif) qui organisent leurs déplacements et les introduisent à la pratique des rites. Il faut donc rester dans son groupe, s'accorder à cette discipline collective qui devrait garantir le bon déroulement du pèlerinage: le groupe de "pays" constitue l'unité d'appartenance et de référence qui permet d'évoluer sans se perdre dans la foule — plus de deux millions de personnes, hommes et femmes vêtus de blanc.

Le premier déplacement, préalable non obligatoire au hajj, mais effectué par tous, conduit les pèlerins sur la tombe du Prophète à Médine, point d'ancrage affectif du sentiment religieux. L'émotion est liée à la personne du Prophète, à ses compagnons (on se rend sur leurs tombes dans les cimetières environnants), à sa famille ( $a b l a l$-nabi: les "gens du Prophète»). Puis, les pèlerins se dirigent vers La Mecque. Avant de franchir l'enceinte de la ville, ils s'arrêtent pour faire leurs ablutions et revêtir l'ibrâm (deux pièces de toile blanche non cousues). Chacun prononce la talbiya (déclaration d'intention qui devient invocation, très fréquemment répétée tout au long du pèlerinage ${ }^{5}$ ): il est alors mubrim, en état de consécration, respectant les interdits vestimentaires, sexuels et de sociabilité. Le pèlerinage proprement dit commence après ces observances accomplies individuellement.

5. «Me voici à toi, ô Dieu. Me voici à toi. Me voici à toi. Tu n'as point d'associé. À toi vont les louanges, de toi viennent la grâce et le souverain pouvoir. Tu n'as point d'associé ». 
La Mecque, «mère des villes» (umm al-qura), renvoie aux fondements de la Tradition abrahamique. C'est le premier des Lieux saints. Ici, les pèlerins revivent, dans l'espace rituel des origines, l'origine même de leur religion. En islam, le sacré réside dans les textes, les paroles et les actes de la Tradition, mais ne se matérialise pas. Cette représentation du monde admet une exception majeure : la Ka'aba est le seul objet du culte islamique. C'est le centre et l'origine de la liturgie musulmane, la «maison de Dieu », la qibla ("direction de la prière») de l'univers. Situé dans l'enceinte de la Grande mosquée, le monument cubique haut d'une quinzaine de mètres porte à son angle méridional la pierre noire ${ }^{6}$ enchâssée par Mahomet pour marquer le point de départ du rite de circumambulation (tawaf), rite qui symbolise le mouvement circulaire de la Terre autour de l'axe polaire hajj signifie littéralement « décrire un cercle».

Les rites préalables consistent en un tawaf de sept tours, une prière et un $s a^{\prime} y$, course entre les lieux dits Safa et Marwa qui s'effectue dans une immense galerie accolée à la Grande mosquée. Le pèlerinage a lieu entre le 8 et le 12 dhu al-hijja, se compose d'une 'umra (visite à la Ka'aba) et d'un ensemble de rites accomplis aux alentours de La Mecque (voir notamment Wensink et coll. 1990, 37-38; Zeghidour 1989, XI). Le 7, un prône (khutba) rappelle aux pèlerins leurs obligations. Le 8 , ils se rendent à Mina, vallée désertique surplombée de rochers, et passent la nuit dans des tentes. Le 9, ils gagnent la vallée de "Arafat, pour le wuquf («station"): devant le jebel al-rahma ( «colline de la miséricorde»), ils demeurent en prière de midi au coucher du soleil; puis, ils se rendent à Muzdalifa pour un wuquf nocturne. Le 10, ils retournent à Mina pour procéder à la lapidation d'un pilier symbolisant le diable et à l'immolation d'un animal, généralement une brebis ou une chèvre, ou encore, pour les plus riches, un chameau. Puis, ils se font raser la tête ou couper les cheveux, et les interdits cessent. Les pèlerins reviennent à La Mecque pour un nouveau tawaf autour de la $K a$ 'aba qui porte ses nouvelles tentures. Du 11 au 12, ils retournent à Mina pour d'autres lapidations, qui peuvent se prolonger jusqu'au 13. Ils reviennent à La Mecque pour le tawaf d'adieu, et éventuellement un nouveau $s a^{\prime} y$. Ils prient devant le mausolée d'Abraham et boivent à la source de Zemzem, achètent aussi cette eau qui contient la baraka. Ils quittent la ville et prennent le chemin du retour.

6. Pierre noire extraite de la montagne par Ismail, ou apportée du paradis par l'ange Gabriel, ou encore tombée du ciel à la façon d'un météorite. 
L'émotion pèlerine est complexe: dans le temps du rite, les pèlerins ressentent la foule à la fois comme une figure massifiée de l'identique — «le pèlerin » par excellence - , une protection nécessaire et un danger potentiel. Le bon déroulement du haji exige cette multitude, dans chaque acte rituel, dans chaque déplacement. Le pèlerinage est une véritable épreuve physique, qui se déroule souvent dans des conditions climatiques difficiles: en été, le wuquf se tient à une température atteignant $50^{\circ} \mathrm{C}$, et les insolations sont nombreuses. La plupart des pèlerins logent à plusieurs dans des chambres sans confort, et ne peuvent prendre aucun repos.

Logement, nourriture, services d'accueil, de portage, d'orientation, transport entre les différents lieux, tout se paye, et tout est très cher. La rétribution du guide, obligatoire, est très élevée. Il faut donner un bakshish pour obtenir dans les temps le laissez-passer pour Médine, mener d'âpres marchandages avec les chauffeurs de taxi et les commerçants. Tout s'achète, même l'eau sur bien des sites, pour boire et pour les ablutions. Ne parle-ton pas de mawsem al-haji, la "saison du pèlerinage ", expression qui sonne bien comme une saison touristique...

Tous ces aspects ont leur place dans les discussions des voyageurs immobiles. Ils partagent les émotions de cette expérience mystique par la prière, par le sacrifice. Le soir, ils se réunissent pour regarder à la télévision les très nombreux reportages consacrés au pèlerinage. Ils apprécient les ors des Lieux saints. Ils admirent la foule dans son importance numérique et sa diversité. Ils plaignent les pèlerins qui peinent à avancer lors du tawaf. Ils commentent les efforts du royaume saoudien pour l'aménagement des sites, le développement des voies de circulation, l'organisation des déplacements, l'efficacité des services d'ordre. Ils sont émerveillés et inquiets, car sur les différents sites, la promiscuité est extrême et entraîne des bousculades parfois mortelles — en juillet 1990, 1426 pèlerins meurent asphyxiés ou piétinés dans un tunnel; en mai 1994, en avril 1998, en mars 2001, en février 2003, plusieurs centaines de personnes meurent pendant le rite de lapidation. Les incendies, très redoutés, font de nombreuses victimes - 343 dans un camp à Mina, en avril 1997. Les accidents de la route sont fréquents.

La mort en pèlerinage préoccupe constamment les voyageurs immobiles. Chaque fois que la télévision annonce un accident mortel à La Mecque, on se lamente et on se réjouit en même temps. Mourir pendant le hajj est en effet un risque, mais la teneur de ce risque est difficilement évaluable, car cette mort-là garantit le paradis. Les parents, les proches et les voisins ne souhaiteraient pas la mort de leur représentant, d'autant moins 
que les croyants ne s'autorisent d'ordinaire aucun «vœu» sur ce sujet. Ils l'envisagent dans cette circonstance singulière comme une dernière volonté, car celui qui meurt pendant le hajj devient hâjj. La différence entre ces deux processus de transformation reste fondamentale: celui qui devient hâij par sa mort en pèlerinage «agit » pour lui et lui seul, alors que celui qui accomplit le pèlerinage devient hâjj pour sa communauté.

Pèleriner, dans la mobilité et dans l'immobilité, atteint ici plusieurs dimensions. Ce voyage rituel particulier se compose bien d'un début, d'un milieu, d'une fin. Son déroulement nécessite des étapes de détachement, d'agrégation, et à nouveau de séparation. Dans le collectif du pèlerinage, chaque individu joue pour son propre compte la même partie que son voisin, au même moment, au même rythme, l'intensité émotive variant bien entendu selon l'histoire et l'identité de chacun. Cependant, le cadre rituel est donné une fois pour toutes, un cadre rituel au sens d'un espace physique et monumental que chacun connaît, même sans l'avoir arpenté personnellement. Le cheminement rituel est connu de tous, mais il est distillé plutôt que fourni d'emblée au pèlerin. Celui-ci est à chaque instant en situation de découverte, d'apprentissage, d'adaptation: chaque action correspond à une "première fois ». Alors que dans le même temps, paradoxalement, le voyageur immobile est beaucoup plus familier des actes rituels qui correspondent pour lui à une suite de situations connues, une sorte de longue répétition. Autrement dit, le pèlerin en situation parle au présent, et le voyageur immobile peut choisir le temps de son récit, passé ou futur. Face au pèlerinage comme rite de passage, l'inquiétude du pèlerin vient de ce qu'il ne sait pas (il est dans une situation d'ignorance analogue à celle du futur «initié»), et le voyageur immobile est inquiet lui aussi, mais parce qu'il sait.

\section{Peindre le pèlerinage}

Un acte particulier des voyageurs immobiles s'inscrit hors ou au-delà du rite: ils se réunissent pour faire exécuter sur la maison du futur hâjj une décoration spécifique. Ces peintures murales de pèlerinage sont assez discrètes en Libye, en Syrie et en Palestine; on en trouve quelques-unes au Nord Yémen et au nord du Soudan. Elles sont beaucoup plus courantes en Égypte, où certaines réalisations sont réellement foisonnantes. Elles se limitent parfois à quelques mots et une date au-dessus de la porte, ou s'étendent sur toute la façade qu'elles recouvrent de représentations éclatantes. 


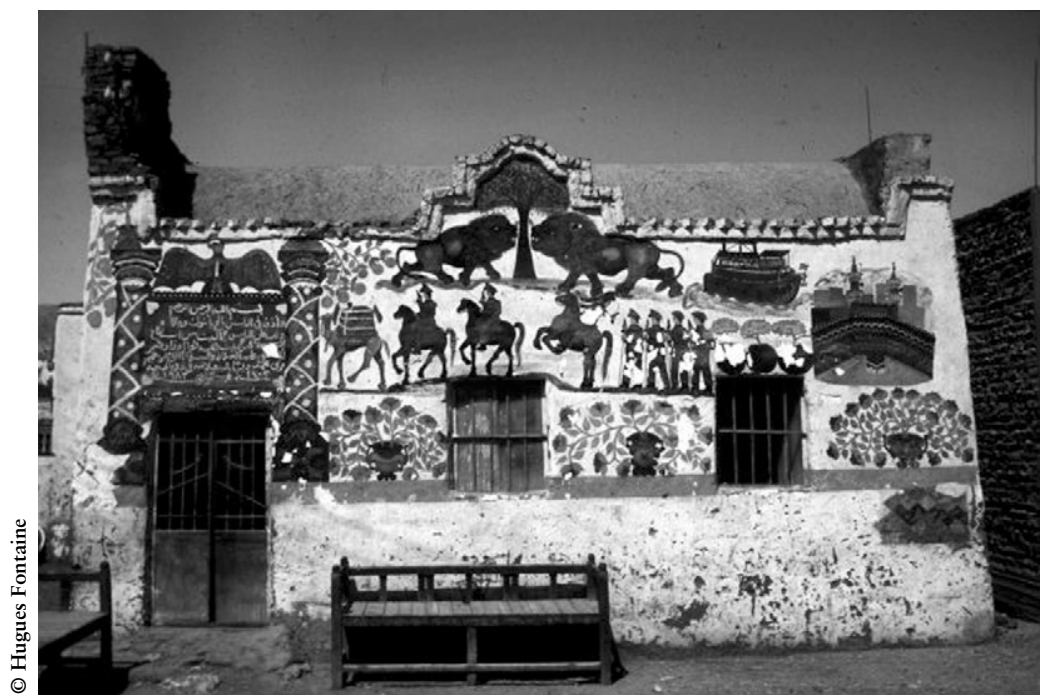

Façade de la maison d'un hâjj ornée de peintures de pèlerinage

Les peintres de pèlerinage, dont l'activité est de fait saisonnière, ne sont ni des professionnels ni des ouvriers en bâtiment ni des artistes. Reconnus comme «spécialistes» par les villageois, ce sont souvent des instituteurs, originaires ou non du village, qui ont manifesté leur goût pour la calligraphie en l'exerçant à la mosquée et à l'école, et ont acquis l'expérience et l'habileté. Ils signent très rarement leur travail sur les murs. Chacun possède un style particulier: en choisissant tel peintre, les commanditaires n'auront pas de (mauvaise) surprise. La décoration fait l'objet d'un contrat entre le représentant du pèlerin (son frère, son fils) et le peintre. L'accord porte sur l'étendue du travail, les matériaux employés, le temps nécessaire à l'exécution, la rémunération subséquente. Face à son ouvrage, le peintre n'est jamais seul: les membres de la famille, les voisins, les passants l'encouragent, scrutent ses gestes, le surveillent. Les peintures sont exécutées sur la façade principale, et très rarement sur les façades latérales ou dans la cour. Tout est fait pour le regard extérieur, celui du passant.

Les peintures sont beaucoup plus fréquentes dans les villages, les bourgs et les villes moyennes que dans les grandes villes. Elles font cependant l'objet d'une discussion récurrente à l'échelle nationale. Chaque année, à l'époque du pèlerinage, les journaux égyptiens participent à l'actualisation de cette tradition. Des journaux populaires nationaux comme les suppléments culturels des journaux cairotes présentent immuablement les mêmes arguments 
fondamentaux. Les peintures de pèlerinage sont assimilées aux rituels d'une Égypte populaire et millénaire: au même titre que les peintures des tombes et des temples de l'Antiquité, les décorations murales actuelles font partie du patrimoine égyptien. En relation avec l'interdit coranique des représentations humaines, les peintures murales sont qualifiées de religieuses et profanes à la fois, par une formule journalistique maintes fois répétée selon laquelle l'islam prohibe les représentations religieuses, mais n'interdit pas l'art profane.

Sans reprendre ici la discussion sur le statut des images en islam, rappelons quelques éléments majeurs de la doxa qui font l'objet d'énonciations et de commentaires sur le terrain égyptien. D'une part, le Coran n'impose pas un strict interdit de figuration, mais jette l'anathème sur l'usage des images, et en particulier des statues. Le texte relate que le Prophète, quand il eut conquis La Mecque, détruisit avec sa canne les 360 idoles que les païens avaient dressées autour de la Ka'aba. L'aniconisme coranique vise l'image de la divinité, celle des envoyés divins (rusul), des prophètes (anbiya) et des saints (awliya): il s'agit de proscrire l'objet d'un culte éventuel pour lutter contre la tentation de l'association du créé à l'incréé. D'autre part, il faut préserver la dignité primordiale de l'homme dont la forme faite "à l'image de Dieu " ne sera ni imitée ni usurpée par une œuvre d'art, une "création » humaine. Des hadiths ( «dits» du Prophète) rappellent qu'au jour du jugement dernier les artistes seront sommés de présenter leurs œuvres sous leur forme vivante. Ces grandes lignes connaissent des adaptations ou des réalisations significatives selon les époques, les régions, les régimes politiques.

La situation locale, singulière et spécifique, donne quelques indices pour appréhender un paradoxe très répandu concernant l'identité et les valeurs sociales. En Égypte, les peintures murales représentent rarement les personnages sacrés, à l'exception notable d'Abraham et de son fils dans la scène du sacrifice. Par contre, il est fréquent que des saints locaux soient photographiés de leur vivant, que leurs images circulent librement y compris après leur mort, vendues lors de la «fête-anniversaire» (mawled) que leur consacrent chaque année leurs descendants et leurs adeptes, pour la plupart réunis au sein des confréries soufies. Il est évident que de telles pratiques tiennent à la dimension populaire de la religion, analogue en bien des aspects chez les musulmans et chez les Coptes, en contexte rural tout particulièrement ${ }^{7}$. En outre, les peintures de pèlerinage ne possèdent pas en propre une valeur sacrée. Ce sont des espaces où se déposent plusieurs niveaux de conscience

7. Toujours à l'échelle locale, de nombreux musulmans rendent hommage à Mari Girgis (saint Georges) lors de la fête annuelle que lui dédient les Coptes. 
du sacré en fonction des images : les origines, le temps de la Révélation, la Maison... Ce sont les lieux d'une certaine mémoire, plutôt que des lieux de mémoire, qui ne se transforment jamais en objets de culte ou de dévotion: on ne touche pas les peintures comme on le fait avec les dessins prophylactiques des temples, on ne leur demande pas la baraka, que l'on peut obtenir auprès du saint vivant, sur la tombe du saint décédé, par contact avec le hâjj lui-même ou avec les objets qu'il a rapportés des Lieux saints.

Les peintures ne sont pas non plus des objets d'art: elles ne sont pas valorisées pour des raisons esthétiques. Les gens qui les réalisent n’ont pas localement un statut d'artiste, et elles sont produites non par un seul individu, mais par la communauté tout entière. Les rares pèlerins qui refusent que leur maison soit peinte, ceux qui repoussent les images, invoquent l'interdit de figuration et disent suivre à la lettre les préceptes de l'islam en reprenant à leur compte le geste du Prophète contre les statues mecquoises.

Cependant, les peintures forment bien un système symbolique, produit, approprié et reproduit par l'ensemble du groupe: un outil de communication, un instrument d'intégration et de valorisation sociales, l'expression de la solidarité entre les individus. Celui qui refuse de faire décorer sa maison alors que tous ses voisins ont satisfait à la tradition, ou celui qui tente d'imposer un style nettement différent, qui tranche totalement avec le style local, celui-là passe pour un original: il se démarque de la communauté, il s'en exclut. L'art figuratif populaire trouve sa place dans la religion populaire. Sa fonction religieuse peut être qualifiée de périphérique: il est avant tout le mode d'expression communautaire d'un sentiment, d'une émotion, d'une vision du monde.

\section{Ce que montrent les murs}

Les murs disent d'abord - et c'est parfois le seul message - le nom du pèlerin, précédé de la mention de son nouveau statut, et l'année du pèlerinage, indiquée par la date du calendrier hégirien et celle, administrative, du calendrier grégorien. S'y ajoutent éventuellement des versets coraniques, des hadiths et des formules votives ${ }^{8}$ propres au pèlerinage qui ont plusieurs

8. Pour cet article, je choisis un traitement différencié des messages écrits. Les versets coraniques sont présentés dans la traduction de Jacques Berque (1995). Les hadiths font l'objet de plusieurs formulations et commentaires de savants musulmans depuis le recueil établi par Mâlik ben Anas (96-179 de l'hégire / 712-795 de l'ère chrétienne), fondateur de l'école malékite. Pour ces « dits » du Prophète comme pour les formules votives, je propose mes traductions issues des discussions menées sur le terrain avec différents acteurs des peintures de pèlerinage. 


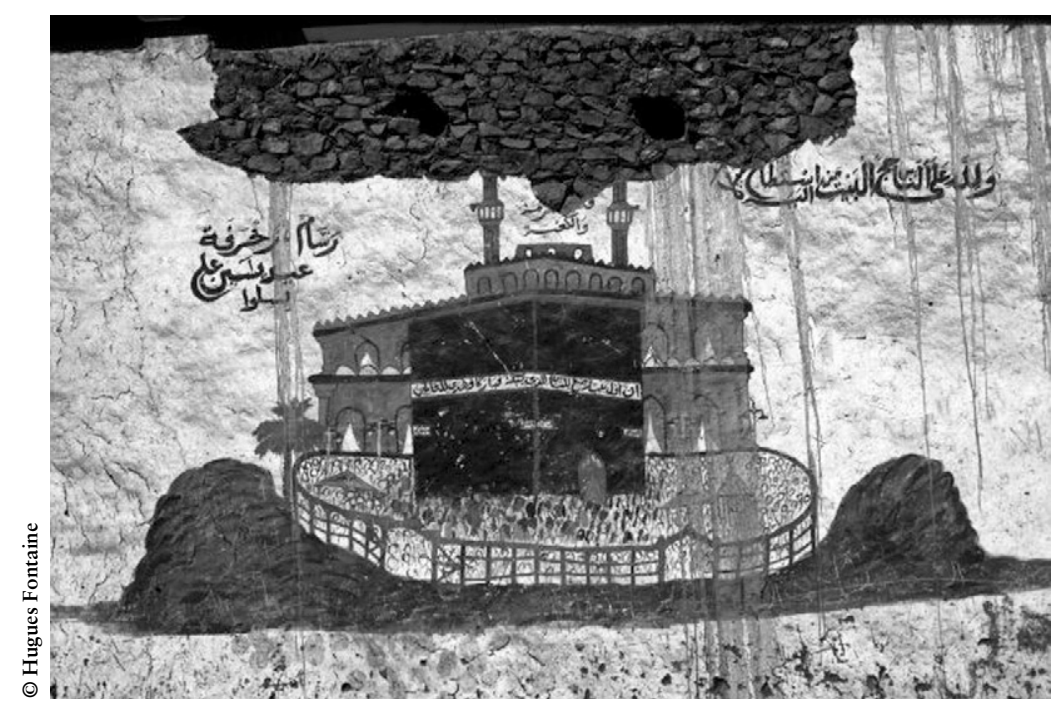

Peinture murale: la Ka'aba, à La Mecque

fonctions. Ils rappellent l'injonction coranique: " pour Dieu, le pèlerinage à la Maison s'impose à quiconque en a la possibilité » (Coran 3,97); "lance parmi les hommes l'appel au pèlerinage: qu'ils te viennent à pied ou sur quelque bête amaigrie " $(22,27)$; "On n'entreprend un long voyage que pour visiter les trois mosquées: la Sainte Mosquée de La Mecque, ma mosquée de Médine et la mosquée d'Al-Aqsa à Jérusalem " (hadith) ${ }^{9}$. Ils assimilent la maison du hâjj à la maison de Dieu: "Entrez avec un salut, en toute sécurité » (Coran 15,46). Ils commentent l'acte: "Il a fait le pèlerinage à La Mecque et la visite au tombeau du Prophète» (hadith); "Pèlerinage bien accompli, fautes pardonnées, sa'y récompensé » (formule); "Le pèlerinage bien accompli n'a d'autre rétribution que le paradis » (hadith); "Celui qui aura accompli quarante prières rituelles dans ma mosquée aura droit à ma protection [le jour du jugement dernier] » (hadith). Ils conseillent le passant: "Quand tu rencontres un hâjj, salue-le, embrasse-le, et demande-lui d'invoquer pour toi le pardon de Dieu, parce que ses péchés ont été absous et que ses prières seront reçues » (hadith). D'autres, enfin, viennent commenter les images des lieux sacrés: «Entre ma tombe et mon minbar, c'est un des jardins du Paradis » (hadith); "Cette ville est sacrée. Dieu l'a rendue sacrée le jour où il créa les cieux et la terre. Elle restera sacrée jusqu'au jour

9. Les hadiths sont entourés de la formule rituelle du Basmala: «Au nom de Dieu, le Clément, le Miséricordieux, [...] a dit le Prophète, que la bénédiction et le salut de Dieu soient sur lui ». 


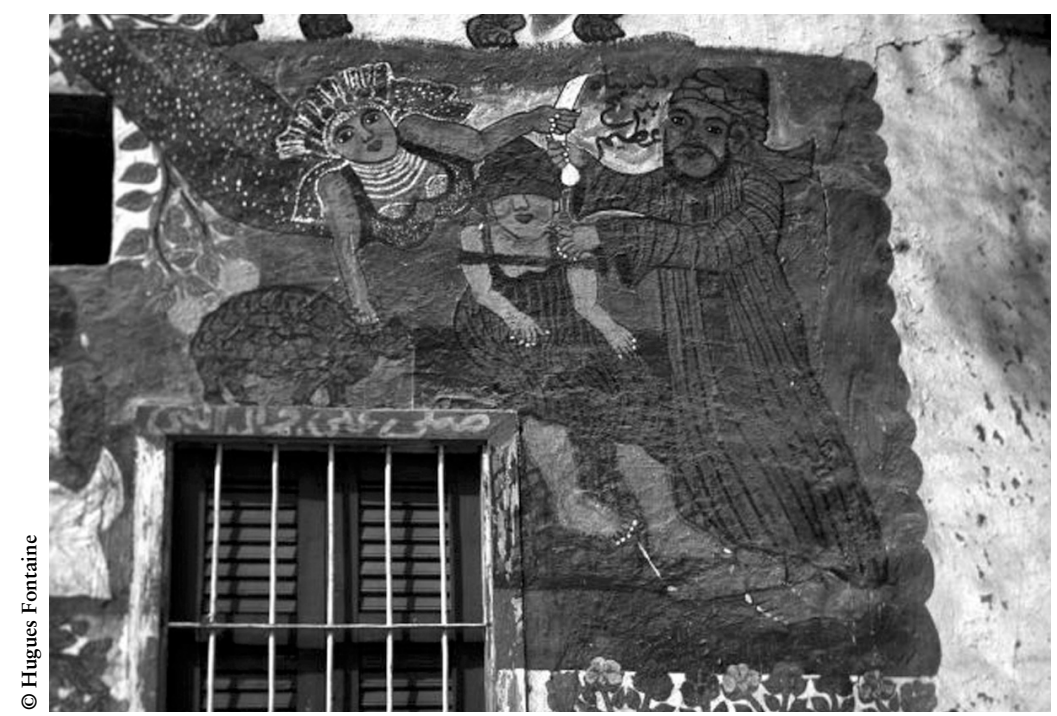

Peinture murale: scène du «sacrifice d'Abraham »

du jugement dernier » (hadith); "La première Maison instituée pour les habitants de Bakka, en bénédiction et guidance pour les univers » (Coran 3,96). Rien n'est dit qui identifie le hâjj au-delà de la mention de son statut, qui le distingue en particulier, et rien ne personnifie l'acte célébré.

Les murs portent très fréquemment les monuments emblématiques des Lieux saints: les mosquées de Médine et de La Mecque, la Ka'aba avec la pierre noire. On y voit des pèlerins en prière, certains sont isolés - aucun n'est identifié par les commentateurs comme le hâjj de la maison peinte d'autres sont réunis en une foule compacte dans les mosquées, à La Mecque pour le tawaf et le $s a$ ' $y$, ou forment une longue procession sur les flancs du Mont 'Arafat. On trouve aussi, quoique très rarement, deux personnages de la Tradition: Bilâl, l'esclave affranchi, premier muezzin de l'islam, et Al-Boraq, le coursier ailé, monture du Prophète lors de son voyage nocturne (isra') entre La Mecque et Jérusalem.

Toutes les phases rituelles ne sont pas bonnes à peindre. Le rite de lapidation n'est jamais représenté. Par contre, le sacrifice et l'offrande d'un animal en souvenir de la victime d'Abraham suscitent de nombreuses images. Ce moment critique est donné à voir dans une suspension du mouvement, les personnages sont tournés vers le spectateur qu'ils regardent, ou prennent à témoin. L'ange Gabriel est ici femelle, il descend du ciel, et arrête à pleine main le fer avec lequel Abraham s'apprête à immoler son fils. 
La victime a les yeux bandés. De sa main droite, Gabriel dépose le mouton, monnaie de l'échange et gage de la miséricorde divine. Une confusion très courante sur l'identité du fils conduit au sacrifice fait dire aux Égyptiens qu'il ne s'agit pas d'Isaac, mais d'Ismail, fils de l'Égyptienne Hagar et considéré comme l'ancêtre mythique des Arabes. Au cours du pèlerinage, le sacrifice consiste en une juxtaposition d'actes individuels et non en une cérémonie collective. C'est pourquoi les représentations murales renvoient au mythe, à l'acte de référence avec les personnages (le père, le fils, l'ange et le mouton), et non au rite, à la communauté. Car il s'agit bien de revivre, commémorer, célébrer le sacrifice originel et fondateur. Dans le même sens, cette scène mythique est souvent reproduite, car elle tire sa force du fait que tous les croyants accomplissent le même sacrifice, où qu'ils soient, en même temps. Dans le déroulement du pèlerinage, c'est le seul moment vécu comme absolument synchrone entre les pèlerins et les voyageurs immobiles qui, ensemble, incarnent alors l'umma, la «communauté musulmane».

Les thèmes évoqués jusqu'ici ne suffisent pas à raconter le pèlerinage, ni sur les murs ni dans les récits. Car le haji ne correspond pas seulement, dans les représentations et les pratiques locales, à un acte religieux dont le signifiant serait susceptible d'agir de manière isolée. Il s'insère, comme toute manifestation mystico-rituelle, dans un dispositif social et culturel localisé. Les peintures murales de pèlerinage mêlent ainsi plusieurs registres iconographiques et symboliques qui rappellent, ou convoquent les valeurs réelles et idéelles de la communauté. Quelques scènes de la vie quotidienne rurale prennent des allures folkloriques désuètes, telles ces paysannes portant des cruches d'eau sur la tête ou sur la hanche. Une flore inventive et envahissante parle d'abondance. Des animaux domestiques, ânes et buffles, côtoient les scorpions emblématiques de la confrérie soufie rifaîya, les félins pharaoniques, les crocodiles du Nil et l'aigle national. La patrie est présente par le drapeau et les soldats en armes. L'évocation du mawled, rassemblement des descendants du saint local et de ses adeptes, en utilise les séquences les plus festives: des musiciens et des chanteurs récitant les gestes fondatrices (Abu Zayd al-Hilali, Antar et Abla), les chevaux et les cavaliers des joutes équestres (mirmah), les adversaires au jeu de bâton (tahtib), autant de souvenirs entretenus des valeureux guerriers bédouins revendiqués comme leurs ancêtres par les populations rurales d'Égypte.

La vie politique ne constitue pas un thème déclinable sur les murs des maisons. Deux exceptions, de natures très différentes, sont toutefois à mentionner. D'une part, Gamal Abdel Nasser, marchant à la tête d'une troupe 


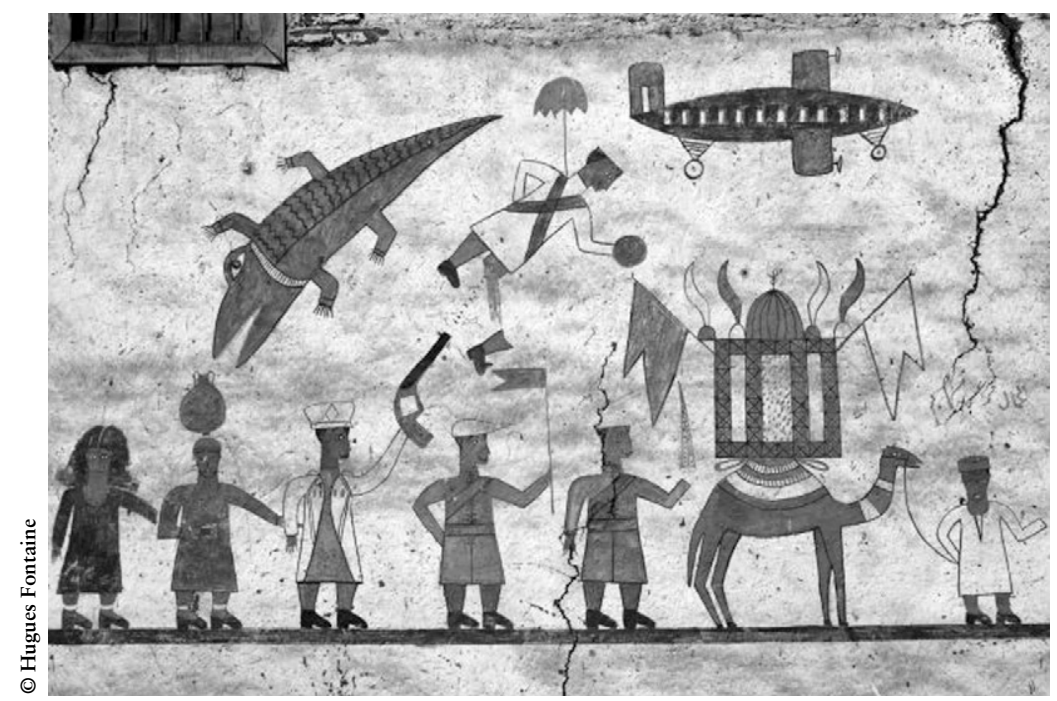

Peinture murale: intervention militaire contre des terroristes à La Mecque

militaire, dominant les soldats de sa haute stature: le président mort en septembre 1970 est encore présent sur les murs peints à la fin des années 1980. D'autre part, des parachutistes en vol, armés de fusils mitrailleurs, ou portant une bombe et blessés pendant leur descente par le tir d'un soldat au sol: cette scène à la fois dramatique et incompréhensible est très appréciée des commentateurs, dont les explications renvoient à l'intervention des gendarmes français contre des terroristes à La Mecque ${ }^{10}$. Alors que des attentats ont été régulièrement perpétrés durant la décennie 1979-1989, seul cet événement s'inscrit sur les murs.

\section{Ce que disent les peintures à propos du voyage}

Le discours des murs peints, disert et divers, s'accorde sur un thème, celui des moyens de transport. La notion même de voyage (safar) est attachée, dans les paroles attribuées au Prophète, aux pèlerinages: "On n'entreprend un

10. Le 20 novembre 1979 ( $1^{\text {er }}$ moharram 1400), 200 terroristes prennent en otage 50000 personnes dans la mosquée de La Mecque. Après un début de négociation et la libération d'un certain nombre de pèlerins, le royaume saoudien fait appel au Groupe d'intervention de la gendarmerie nationale (GIGN) français. Le 23 novembre, trois gendarmes et le capitaine Barril sont «convertis » à l'islam lors d'une brève cérémonie. Les gendarmes concluent leur intervention en utilisant des gaz de combat. 
long voyage que pour visiter les trois mosquées: la Sainte Mosquée de La Mecque, ma mosquée de Médine et la mosquée d'Al-Aqsa à Jérusalem » (hadith). Le chemin de La Mecque reste dans la mémoire populaire une épreuve périlleuse: longues journées de chaleur et de soif sur les pistes du désert, attaques des Bédouins, etc.

Depuis le XVI ${ }^{\mathrm{e}}$ siècle, la caravane égyptienne escortait le mahmal, palanquin de bois qui symbolisait l'autorité politique nationale, et les tentures de la $K a$ ’aba, offertes chaque année par l'Égypte. Avant le départ, le palanquin était présenté à la population du Vieux Caire lors de grandes processions de la place de la Citadelle à la mosquée de Sayyedna Hussein. Les autorités remettaient la longe du chameau qui portait le palanquin à l'amir al-hajj, littéralement le "chef du pèlerinage ", le responsable de la caravane. Du Caire à Médine, puis à La Mecque, le voyage durait environ 35 jours. Au retour, une foule nombreuse se pressait pour toucher les objets qui avaient traversé les Lieux saints, pour en obtenir la baraka. À la suite de la prise du pouvoir par Ibn Sa'oud en 1924, l'ampleur des fêtes du Caire déclina. Le cortège ne comptait plus que sept chameaux: le premier transportait le mahmal, le second son drapeau, le cheikh de la caravane montait le troisième, et suivaient quatre musiciens, jouant du tambourin et de la flûte. Les pèlerins égyptiens continuèrent de se regrouper à Djedda autour du palanquin qui y était transporté par bateau jusqu'en 1926. Les changements politiques de 1952 ont mis un terme à la tradition: le mahmal du roi Fouad, conservé au Musée d'ethnographie de la Société de géographie du Caire, n'est plus qu'un vestige de l'ancien régime. La caravane du pèlerinage est donc un lointain souvenir et les peintures la situent bien dans le passé. Le chameau porteur de palanquin représenté sur les façades est souvent décrit comme la monture du Prophète ou celui du mahmal de la caravane; mais il est plus souvent identifié par les gens au chameau des cortèges du mawled.

Aujourd'hui, moins de deux heures d'avion séparent Louxor de Djedda. C'est le moyen de transport qu'empruntent la plupart des pèlerins, mais certains en Haute Égypte continuent de se rendre par le train jusqu'au port de Safaga pour traverser la mer Rouge en bateau. Les peintures de pèlerinage mêlent tous les moyens de transport, anciens et modernes: elles ne racontent jamais le voyage d'un hâji particulier sur un mur particulier. À côté du chameau transportant le mahmal, on voit l'âne familier du paysage villageois, le cheval de fête ou de défilé militaire, la charrette, la calèche, la voiture, l'autobus, le taxi, le train. Les bateaux ressemblent peu aux navires touristiques 
ou aux felouques qui croisent sur le Nil, mais plutôt à des cargos chargés de conteneurs. Les avions aussi provoquent l'imagination des peintres: on peut suivre à travers les ruelles des villages égyptiens l'évolution de l'aéronautique, du bimoteur au jet à réaction, jusqu'à l'avion-radar AWACS.

\section{Retour de pèlerinage}

L'émotion du retour est plus impressionnante encore que celle du départ. Les parents et les amis se regroupent plusieurs jours à l'avance: chacun connaît la date à laquelle reviendra celui qu'il attend, mais l'attente en commun fait partie du rituel. Les policiers maintiennent la foule à l'extérieur d'une enceinte réservée aux officiels. À chaque arrivée d'un autobus de l'aéroport, les haut-parleurs diffusent le nom des voyageurs et les familles se précipitent dans la confusion, franchissant les barrières, échappant au contrôle. Embrassades, larmes, cris, premières bénédictions : ils sont soulagés, heureux de se retrouver, et le manifestent publiquement.

Ces démonstrations collectives sont rares en Égypte, où l'expression des affects est d'ordinaire très codifiée, retenue par une profonde pudeur, masquée par la litanie des formules usuelles. La joie des retrouvailles, l'émotion, l'admiration et le respect se lisent dans les yeux de chacun. Les pèlerins sont entraînés vers les voitures, leurs habits blancs disparaissant au milieu des galabeyyât (sing. gallabeyya) colorées de ceux qui les accueillent. Sous la pression des parents, certains ouvrent leurs bagages et commencent en public - contrairement à toutes les règles de l'échange - la distribution des cadeaux investis de la baraka des Lieux saints. L'inventaire est sans fin: foulards, tissus, ombrelles, parfums, eau de Zemzem, appareils électroménagers et radiocassettes achetés en zone franche, fruits, biscuits, café, tapis de prière, affiches pieuses, jusqu'au certificat de pèlerinage signé par quatre témoins, et la carte de visite du guide.

Dans les voitures qui repartent, le pèlerin répond déjà aux questions des siens. Au village, il découvre les peintures et les visites commencent: salutations, félicitations, souhaits de bienvenue et de bonheur qui rejaillissent sur la famille, les voisins, la communauté. Tous viennent partager sa joie, participer à sa consécration sociale. La dépense cérémonielle se poursuivra jusqu'à ce que chacun ait obtenu ce que son rang, son degré de parenté ou de proximité, lui fait espérer : nourriture et boisson, objets et paroles.

Le récit de pèlerinage (ribla hijaziya) est un grand moment. Discours à la fois mystique et réaliste, il témoigne de l'expérience religieuse et de l'émotion du voyage. L'auditoire est attentif aux moindres détails: des heures 
durant il exige du conteur, tour à tour serein et illuminé par la force du souvenir, qu'il habille la trame rituelle connue de tous par sa propre expérience. Certains épisodes sont répétés. Le récit porte moins sur le pèlerinage que sur les émotions collectives, des moments particuliers que chaque auditeur peut reprendre à son compte, en s'imaginant dans la peau du personnage.

En écoutant le récit du hâjj, en y participant par leurs commentaires et leurs exclamations, les voyageurs immobiles achèvent leur propre voyage. C'est par le partage impossible de l'émotion du pèlerinage qu'ils atteignent les limites de leur propre expérience. Ils vont maintenant participer à la dernière étape du voyage du pèlerin en aidant à sa réintégration dans la communauté.

\section{Le titre et le statut de hâaj}

Tout au long de son itinéraire, le pèlerin est à la fois l'acteur individuel d'une transformation personnelle et le représentant du groupe dans un phénomène de transformation collective. Sa communauté l'a exclu, il a rejoint le collectif du pèlerinage au sein duquel et grâce auquel il a acquis son nouveau statut. Il revient maintenant prendre une nouvelle place. Celui ou celle qui a accompli le pèlerinage porte désormais le titre de hâjj ou hâjja. C'est la signification du terme, dans la simplicité et la force de l'expression. Mention honorifique certifiant l'acte de foi, l'appellation prime désormais toute autre: elle précède le nom sur les documents officiels. Le titre de hâjj prévaut aussi sur tous ceux acquis dans l'exercice d'une profession: le commissaire de police qui a fait le pèlerinage est hâajj avant tout, comme le commerçant qui modifie son enseigne dès son retour des Lieux saints. Pour les hommes politiques aussi, c'est une affaire de statut, la notoriété étant consolidée par le voyage à La Mecque.

Au sein de la famille, le titre tend à remplacer les termes de parenté qui expriment en même temps le respect: l'oncle paternel, 'amm, se trouve encore grandi dans l'estime que lui porte sa famille quand il a accompli le pèlerinage. Pour une femme, le titre de hâjja réfère uniquement au registre religieux et vient renforcer l'expression du respect que l'on doit à une femme âgée et pieuse, mais il porte rarement une connotation sociale. Dans la rue, on s'adresse à un homme âgé, même inconnu, auquel on doit le respect comme à son oncle paternel, en employant le terme de parenté. Mais si sa prestance, sa démarche, son habit, son port de tête donnent le moindre indice de l'éventualité qu'il ait accompli le pèlerinage, on n'hésitera pas à l'appeler hâjji: on lui accordera le prestige, car il en a l'apparence. 
L'usage des titres, comme celui des superlatifs, relève en Égypte d'une forme d'humour populaire très répandue: on interpelle un homme vêtu à l'occidentale, en complet-veston, par un Ya doctor ( «Ô, Docteur!»), un mécanicien en bleu de travail par Ya mohendiss ( (Ô, Ingénieur!»), un personnage imposant au portefeuille rebondi comme son ventre par $\mathrm{Ya}$ Bey (« Ô, mon Prince!»). Ces appellations traduisent un certain goût pour la dérision, jouant sur une subordination feinte dans bien des cas. Le titre de hâjj traduit toujours la déférence, sauf dans le cas suivant: on appellera hâjj le meskin ("pauvre homme»), le marginal ou le fou — celui dont le comportement dépasse les bornes et les normes. Il s'agit alors d'une forme de plaisanterie, à connotation religieuse toutefois, car les fous sont les protégés de Dieu. Ce qui est d'autant plus significatif que l'accès au statut de hâjj vient très souvent couronner l'ascension sociale, pour un homme. Il est courant que le hâjj cumule plusieurs fonctions de premier plan dans la communauté : notable influent, spécialiste en matière de religion, responsable politique local, dirigeant d'une association, etc. Le statut acquis en remplissant son devoir religieux vient consolider sa position au sein de hiérarchies sociales déjà fortement soulignées. Il s'inscrit dans le dispositif de distinction sociale comme un signe nettement valorisé en Égypte, en ville comme à la campagne, et dans l'ensemble de la population.

Devenir hâjji, c'est atteindre un statut social que rien ne modifiera, dont personne ne mettra en question ni la légitimité ni la pérennité. Les murs peints, en revanche, indiquent un rapport totalement différent au temps individuel, collectif et social.

\section{Les peintures et la mémoire, rencontre du rite et de la tradition}

Bien qu'exécutées avec soin, et sujets d'une grande fierté, les peintures de pèlerinage ne sont pas faites pour durer. Elles ne sont pas protégées, ni entretenues, ne font jamais l'objet d'un nettoyage ou d'une restauration. Le temps passant, elles prennent la poussière, s'effacent partiellement sous les traînées de pluie, se dégradent sous l'effet de la sécheresse, se réduisant finalement à une série de taches de couleurs à peine perceptibles dispersées sur la façade - le seul motif valable de renouvellement reste bien sûr un autre pèlerinage effectué par l'un des habitants de la maison.

Les peintures étant ainsi destinées à s'estomper, puis à disparaître, les murs ne sont donc pas peints dans le but immédiat de garder un souvenir ou de fixer une mémoire. L'éphémère de l'existence de ces peintures indique un rapport au temps très singulier. Il s'agit de garder la mémoire d'un événement 
sur un temps court, et c'est justement cette temporalité répétée, tout comme la reproduction de la pratique, qui permet la constitution de cette mémoire atemporelle, la perpétuation de la tradition. Autrement dit, les murs peints proposent des repères temporaires dans le cadre d'un récit élaboré collectivement pour durer sous des formes et des modalités successives, et dont les acteurs singuliers forment un collectif singulier, le communautaire. Les peintures, en tant que support mémoriel, présentent deux messages, l'un explicite (untel a effectué le grand pèlerinage à La Mecque) et l'autre décalé, sinon implicite, et qui doit faire l'objet d'une association pour être décrypté.

Je démontrerai ici cette assertion à partir de l'écrit. L'écrit est un élément essentiel de la religion, puisqu'il transmet avant tout le Verbe, la Parole de Dieu. C'est le véhicule du savoir et de la connaissance, dont la maîtrise et l'usage participent à l'organisation hiérarchique de la société. La majorité des ruraux en Égypte sont illettrés : la plupart des passants ne lisent donc pas les textes religieux reproduits sur les façades peintes. Ils reconnaissent les phrases en tant que formules, et ils les distinguent du langage populaire, dialectal, qui est un mélange vivant de l'arabe classique et de l'image. L'exercice de la lecture est remplacé par celui de la récitation de formules mémorisées, parce qu'elles participent du bagage religieux minimal: les passants associent des éléments techniques, comme la calligraphie et l'emplacement d'une phrase par rapport à une image, et des éléments cognitifs émanant de la croyance et du discours religieux. De cette double association entre l'écrit et l'image, entre la représentation et la croyance, émane la fonction des murs peints comme support de mémoire. Cette association entre le texte et la représentation définit un certain type de construction (ou de structure) mémorielle, mais ne détermine pas la nature du contenu de cette mémoire collective portée par les façades.

Pour comprendre plus précisément la relation entre la mémoire et les peintures, il faudrait interroger le contenu informatif de ces dernières. Or, l'investigation est rapide, car le message est des plus simples: untel a effectué le grand pèlerinage à La Mecque. C'est une nouvelle importante, fondamentale, mais/et que tout le monde connaît déjà. La part informative est sans valeur réelle. Il ne s'agit pas d'information, mais d'attestation : les images attestent, sur la partie la plus exposée de la maison, la véracité et la réalité de l'acte. Cependant, cette attestation n'est pas indispensable, puisque toutes les maisons de hâjj ne sont pas décorées.

Les murs peints n'informent de rien, mais ils commémorent quelque chose, d'une manière très particulière. L'exécution des peintures, dans le 
temps du pèlerinage, en l'absence du pèlerin, est un acte de commémoration décalé dans le temps. Les peintures sont réalisées pendant l'acte qu'elles sont censées commémorer, pendant le rite de passage, avant que la transformation rituelle n'ait abouti - ce qui prouve qu'elles y participent. En ce sens, leur exécution peut être interprétée comme un geste magico-rituel pour assurer le bon déroulement du pèlerinage, la protection du pèlerin, son retour sain et sauf. Les peintures constituent en fait des actes prémonitoires, par lesquels la commémoration bouleverse le temps en opérant avant l'acte, voire en même temps, et non après. Cette confusion des temps entre l'avant, le pendant et l'après, entre le réel et le virtuel, confirme le sens rituel de cette pratique picturale: ce jeu sur le temps suggère alors un mode spécifique de la construction mémorielle et une fonction particulière de la mémoire.

\section{Rite et absence, quelques propositions}

Reprenons maintenant certains aspects de la relation d'échange entre les voyageurs, mobiles et immobiles. Par l'intermédiaire des peintures, les voyageurs immobiles sont introduits dans l'aventure personnelle du hâjj: en contrôlant les images et les paroles, c'est-à-dire les messages exposés sur les murs, ils démontrent que les peintures concernent la communauté et que le pèlerinage d'un seul les concerne tous - les murs disent ce que la communauté veut dire d'elle-même, ce qu'elle veut afficher. Les membres de la communauté participent à la transformation d'un lieu privé (la maison du pèlerin) en un espace commun, l'espace d'une expression publique qui ne peut être que répétitive, contrainte entre le mythe et le rite. Dans ce cadre, toute personnalisation de l'aventure associe nécessairement l'image et le récit, de même que la singularisation d'un pèlerinage nécessite l'association de la connaissance du rituel et du détail d'un événement temporel. Le temps passant, le pèlerinage ne se dit plus au singulier, mais au pluriel. Car, je l'ai démontré, les murs ne racontent pas une histoire personnelle, avérée, et ne correspondent pas à une version «imagée» d'un récit de pèlerinage. Les murs mettent en scène la relation entre la religion et la société : ils affichent en quelque sorte le pacte social. Dans ce sens, ils indiquent l'importance de la répétition, de la reconduction du pacte par la reproduction du rite. Ce que nous disent les peintures, c'est l'importance du rituel dans la reproduction sociale, en tant qu'instrument à double face, individuelle et collective.

Peindre des murs équivaut pour la communauté à prendre une place active dans le rite de passage d'un individu singulier: en participant ainsi 
à la transformation d'un seul, la communauté se transforme elle-même. Dans les faits, elle compte un hâjj de plus, mais ce compte n'a pas réellement d'importance, car la religiosité n'est pas une valeur socialement compétitive qui permettrait de classer hiérarchiquement et relativement plusieurs unités villageoises. La religiosité collective est un état de fait sur lequel les individus ne sont pas censés agir directement - ils n'en ont pas le pouvoir. Par contre, l'engagement de la communauté dans l'événement du pèlerinage participe d'une démonstration essentielle, celle de son existence sociale en tant que groupe, celle de son adéquation à la norme. Il s'agit bien d'un élément constitutif de l'identité communautaire.

Peindre des murs correspond à une étape rituelle qui se déroule en l'absence du pèlerin. Cette absence, qui autorise la participation de la communauté au rite, peut être rapprochée de l'absence temporaire des initiés lors des rites de passage. Si l'on repère dans l'événement social qui nous intéresse ici la structure ternaire du rite, on peut dégager plusieurs séquences parallèles associant la séparation, la latence et l'agrégation. Plusieurs acteurs participent donc aux différentes séquences. Dans un premier temps, la communauté prépare son futur pèlerin et s'en sépare en le confiant au groupe de pèlerins: le futur pèlerin perd son statut de membre de sa communauté (tout en restant son représentant) et, ce faisant, prend le rôle de l'absent social, dont il ne se départira qu'à son retour quand, accueilli et fêté par les siens, il prendra sa nouvelle place dans sa communauté. Dans un deuxième temps, la transformation de l'individu s'effectue dans un groupe de même statut, statut précaire qui s'ouvre pour tous sur l'acquisition du titre de hâjj. La communauté se manifeste et se transforme en l'absence du pèlerin; le pèlerin rejoint un groupe précaire qui ne se forme que dans le but de sa transformation, et disparaît.

L'individu vit donc l'enchâssement de deux processus rituels. D'une part, il quitte sa communauté (première phase du rite principal), et revient avec un nouveau statut (dernière phase du rite principal). Pendant son absence, les villageois jouent la partie collective qui concoure à la constitution de sa nouvelle personnalité: son absence détermine le jeu des autres. D'autre part, il rejoint le groupe des pèlerins (première phase du rite secondaire), s'y intègre, accomplit dans ce collectif les rites qui permettent sa transformation individuelle, puis sort de ce groupe (dernière phase de ce rite secondaire) avec un nouveau statut. Cette exclusion finale, par la désintégration du groupe des pèlerins, permet sa réintégration dans sa communauté. Ces deux rites corrélés sont synchrones de la transformation de la communauté, à la fois collectif rituel et espace rituel. 
Interroger ces pratiques du pèlerinage comme rite de passage amène à considérer le pèlerinage comme un complexe rituel dans lequel acteurs et spectateurs partagent et échangent leurs places et leurs rôles. L'absence des uns rend possible l'action des autres: le processus dans son ensemble nécessite des moments d'absence. Dans le temps du pèlerinage, le sacrifice est de ce point de vue un moment particulier : ce geste rituel ordinaire acquiert un sens initiatique quand il est ainsi partagé, "par les présents et par les absents »; c'est le seul moment rituel qui requiert la présence — virtuelle, factice - de toute la communauté, dans une forme de transcendance spatiale. Et ce n'est pas la seule ambiguïté constitutive et efficace de l'expérience, puisque ces voyageurs immobiles connaissent, vivent, ressentent, perçoivent le rituel sans l'effectuer, de sorte qu'ils pèlerinent sans pèleriner.

\section{Références}

AL-GHazAli, A.H. (1955), «Les secrets du pèlerinage », dans Ih'ya 'ouloûm ed-din, ou Vivification des sciences de la foi / traduction et notes de G.H. Bousquet, Paris, Besson.

Berque, J. (1995) [1990], Le Coran. Essai de traduction de l'arabe, annoté et suivi d'une étude exégétique, Paris, Albin Michel.

Wensink, A.J., J. Jomier et B. LewIS (1990²) [1965], «Hadjdj», Encyclopédie de l'Islam, Leiden / Paris, Brill, vol. 3, p. 33-40.

ZEghidour, S. (1989), La vie quotidienne à La Mecque de Mahomet à nos jours, Paris, Hachette.

\section{Résumé}

Cet article aborde le rite de pèlerinage majeur de l'islam, le grand pèlerinage à La Mecque, par l'observation conjointe des dimensions individuelle et collective. Celui qui accomplit le hajj connaît une expérience unique dont il revient transformé. La communauté villageoise facilite son départ, vit le rituel par procuration, réintègre le pèlerin à son retour. En son absence, les «voyageurs immobiles» ornent de peintures la façade de sa maison, célébrant l'acte de piété, exposant leurs valeurs, affichant le pacte social. L'analyse des engagements individuel et collectif fait apparaitre que la logique du rite de passage est plurielle, relative aux configurations multiples de la présence et de l'absence, du rôle et du statut, du temps et de l'espace, de l'expérience et de l'émotion. 


\section{Abstract}

This article looks at the main Islamic pilgrim rite, the great pilgrimage to Mecca, by taking into account how it is seen by both the individual and the group. The pilgrim who actually partakes in the hajj undergoes what can be considered a unique experience from which he returns transformed. The community facilitates his departure, as well as shares in the ritual and reunites with him when he returns. While the pilgrim is away these "stationery travelers" decorate the front of his house with paintings celebrating the act of piety, exhibiting their values and also their social pact. The analysis of the individual and collective involvement reveals that the logic of this rite of passage has many meanings regarding presence and absence, of role and status, of time and space, of experience and emotion. 